\title{
Association Study between Serum Triglyceride Level and Glucose and Lipid Metabolism in Patients with Polycystic Ovary Syndrome
}

\author{
JING TANG, SHAO JUN. WU* AND HUA QIAN \\ Department of Obstetrics and Gynecology, Taizhou People's Hospital, No. 399 South Hailing Road,Taizhou, Jiangsu 225300, \\ China
}

Tang et al.: Association study in patients with polycystic ovary syndrome

\begin{abstract}
To investigate the serum triglyceride levels and their correlation with glycolipid metabolism in patients with polycystic ovary syndrome. 108 patients who were diagnosed with polycystic ovary syndrome for the first time at the Department of Obstetrics and Gynecology of our hospital from May 2018 to June 2019 were collected. They were divided into an obesity group $(n=64)$ and a non-obesity group $(n=36)$ according to body mass index and a control group of 36 healthy medical examiners with body mass index similar to those of the patients during the same period was selected. The glucose and lipid metabolism levels of the participants were measured by oral glucose tolerance test and insulin release test. Serum triglyceride levels were significantly higher in the obesity and non-obesity groups than in the control group $(\mathbf{p}<\mathbf{0 . 0 5})$. The serum triglyceride levels in the obesity and non-obesity groups were significantly higher than those in the control group $(p<0.05)$ and the obesity group had the highest triglyceride content. The glucose metabolism level in the non-obesity group was significantly higher than that in the control group $(\mathbf{p}<\mathbf{0 . 0 5})$. The Homeostatic model assessment-beta in the control group was significantly higher than that in the obesity group and the non-obesity group, the obesity group had the lowest Homeostatic model assessment-insulin resistance; the Homeostatic model assessment-insulin resistance in the control group was significantly lower than that in the other two groups and the obesity group had the lowest Homeostatic model assessment-insulin resistance $(\mathbf{p}<\mathbf{0 . 0 5})$. Serum triglyceride levels in patients with polycystic ovary syndrome can reflect changes in glucose and lipid metabolism and triglyceride as an inexpensive and routine test can be considered as a surrogate indicator of insulin resistance in non-obesity patients with polycystic ovary syndrome.
\end{abstract}

Key words: Polycystic ovary syndrome, triacylglycerol, glucose lipid metabolism, insulin resistance

The clinical manifestations of polycystic ovary syndrome (PCOS) ${ }^{[1]}$ mainly include hyperandrogenism, ovulation disorder, luteinizing hormone (LH) level increase and relative estrogen deficiency, often accompanied by varying degrees of abnormal glucose and lipid metabolism, such as impaired islet function, arterial atherosclerosis, metabolic syndrome etc. As a common endocrine and metabolic disorder, the incidence rate of PCOS in women of childbearing age is about $6 \%$ to $10 \%{ }^{[2]}$ and about $50 \%$ to $70 \%{ }^{[3]}$ in anovulatory infertility. Dyslipidemia is the most common metabolic abnormality in patients with PCOS. The degree and nature of dyslipidemia are variable and they are closely related to obesity, insulin resistance, cardiovascular disease, etc. ${ }^{[4-6]}$. Triglyceride (TG), as an important indicator to reflect the status of lipid metabolism, the research on TG during pregnancy is mostly limited to the influence of TG on pregnancy outcome $^{[7]}$. So far, there are limited studies on the effect of TG on dyslipidemia and glycolipid metabolism in PCOS patients. Therefore, the purpose of this study is to study the correlation between serum TG and glycolipid metabolism in patients with polycystic ovary syndrome. A total of 108 patients (18-36 y old) diagnosed with PCOS in our hospital from May 2018 to June 2019 were collected for study. The patients were divided into obesity group $(n=64)$ and non-obesity group ( $n=36)$ according to BMI (body mass index) and 36 healthy people with similar BMI were selected as control group. The diagnostic criteria of PCOS were in line with the PCOS diagnostic criteria recommended by the European Society of human reproduction and Embryology (ESHRE) and the American Society of reproductive medicine (ASRM) at the Rotterdam 
conference in the Netherlands in $2003^{[8]}$. Inclusion criteria: the obesity group and non-obesity group were confirmed to have polycystic ovary syndrome by examination; the blood lipid, blood glucose, blood pressure, serum sex hormone and menstrual volume of healthy people were normal. Exclusion criteria: combined with other organic and endocrine diseases; taking hormone drugs. This study was approved by the hospital ethics committee, patients and their families agreed and signed informed consent. Oral glucose tolerance test (OGTT) and insulin release test (IRT): 7.5 pieces of $50 \%$ glucose injection $(20 \mathrm{ml} /$ tube, $10 \mathrm{~g})$ were diluted to $300 \mathrm{ml}$ with water and blood glucose and insulin levels were measured at $30 \mathrm{~min}, 60 \mathrm{~min}$, $120 \mathrm{~min}$ and $180 \mathrm{~min}$ after administration of glucose solution. In addition, the homeostasis index of insulin resistance (HOMA- $\beta=$ fins $\times 20 /($ fasting plasma glucose test (FPG)-3.5); Homeostatic model assessmentinsulin resistance $($ HOMA-IR) $=\mathrm{FPG} \times$ fins/22.5) was calculated by automatic biochemical analyzer. The serum triglyceride (TG), total cholesterol (TC), high density lipoprotein (HDL) and low density lipoprotein (LDL) were measured. SPSS 22.0 software was used for statistical analysis. The normal distribution measurement data is expressed by $\mathrm{x} \pm \mathrm{S}$ and the counting data is expressed by percentage and cases. Independent sample t test was used to compare the two groups, chi square test was used to compare the rate and Pearson correlation analysis was used for single factor analysis. $\mathrm{p}<0.05$ was considered to be statistically significant. As shown in Table 1, the serum TG level of patients in obesity and non-obesity groups were significantly higher than that of control group $(\mathrm{p}<0.05)$ and serum TG level of obesity group was significantly higher than that of non-obesity group ( $p<0.05)$. The level of glucose metabolism in non-obesity group and obesity group was

TABLE 1: COMPARISON OF SERUM TG LEVELS AMONG THE THREE GROUPS

\begin{tabular}{lcc}
\hline Group & $\mathrm{n}$ & $\mathrm{TG}$ \\
\hline Non-obesity group & 36 & $1.15 \pm 0.12$ \\
Obesity group & 64 & $156 \pm 0.17$ \\
Control group & 36 & $0.81 \pm 0.10$ \\
$\mathrm{~F}$ & $/$ & 4.787 \\
$\mathrm{p}$ & 1 & $<0.05$ \\
\hline
\end{tabular}

significantly higher than that in control group $(\mathrm{p}<0.05)$ and that in obesity group was significantly higher than that in non-obesity group $(p<0.05)($ Table 2$)$. As shown in Table 3, the levels of LDL-C, free fatty acid (FFA) and TC in non-obesity group and obesity group were significantly higher, while HDL-C level was significantly lower than that in control group $(p<0.05)$. The levels of LDL-C, FFA and TC in obesity group were significantly higher than those in non-obesity group $(\mathrm{p}<0.05)$ and HDL-C level in obesity group was significantly higher than that in non-obesity group $(p<0.05)$. The results showed that the HOMA - $\beta$ of the control group was significantly higher than that of the obesity group and the non-obesity group and the lowest in the obesity group; while the HOMA-IR of the control group was significantly lower than that of the other two groups, with the lowest in the obesity group ( $<<0.01)$, as shown in Table 4. Through data analysis, we found that serum TG level was directly related to glucose and lipid metabolism indexes $(\mathrm{p}<0.05)$, as shown in Table 5. The main clinical manifestation of polycystic ovary syndrome (PCOS) is metabolic dysfunction of multiple systems, which is more than that before and after puberty. In recent years, the concept of treatment has also changed, from simple ovulation induction and pregnancy in the reproductive period to paying more attention to the relationship between PCOS and long-term diabetes, cardiovascular metabolic diseases, endometrial cancer and so on. Therefore, it is very important to find the metabolic abnormality of PCOS as soon as possible ${ }^{[9]}$. There is a relationship between atherosclerosis, abnormal metabolism of filariase and PCOS. Early detection of abnormal glucose metabolism is of great significance to improve the patient's physical condition and quality of life, but there is still no particularly accurate method to detect insulin resistance ${ }^{[10]}$. In the past decades, the most accurate method was glucose clamp test, but due to its complex operation, time-consuming and labor-consuming, it is rarely used in clinical practice. At present, homeostasis model of insulin resistance (HOMA-IR) is often used as an index to evaluate insulin resistance ${ }^{[1-14]}$. In this study, HOMA-IR was used as an index to evaluate insulin resistance and BMI was used as an index to

TABLE 2: COMPARISON OF GLUCOSE METABOLISM AMONG THE THREE GROUPS

\begin{tabular}{lccccc}
\hline Group & $\mathrm{n}$ & $\mathrm{GLU} 2 \mathrm{~h}(\mathrm{nmol} / \mathrm{L})$ & FPG $(\mathrm{mmol} / \mathrm{L})$ & INS2 $\mathrm{h}(\mathrm{Miu} / \mathrm{L})$ & FINS $(\mathrm{mIU} / \mathrm{L})$ \\
\hline Non-obesity group & 36 & $7.02 \pm 0.61$ & $5.19 \pm 0.37$ & $70.15 \pm 6.38$ & $10.87 \pm 1.17$ \\
Obesity group & 64 & $7.89 \pm 0.73$ & $5.66 \pm 0.56$ & $122.18 \pm 13.44$ & $21.09 \pm 2.25$ \\
Control group & 36 & $5.81 \pm 0.62$ & $4.75 \pm 0.31$ & $37.40 \pm 5.08$ & $4.40 \pm 0.21$ \\
F & $/$ & 6.571 & 5.732 & 12.094 & 7.365 \\
p & $/$ & $<0.05$ & $<0.05$ & $<0.05$ & $<0.05$ \\
\hline
\end{tabular}


TABLE 3: COMPARISON OF LIPID METABOLISM AMONG THE THREE GROUPS

\begin{tabular}{lccccc}
\hline Group & $\mathrm{n}$ & HDL-C & LDL-C & FFA & TC \\
\hline Non-obesity group & 36 & $1.27 \pm 0.11$ & $2.64 \pm 0.26$ & $798.57 \pm 84.29$ & $4.87 \pm 0.42$ \\
Obesity group & 64 & $1.06 \pm 0.12$ & $2.97 \pm 0.32$ & $927.35 \pm 94.20$ & $5.49 \pm 0.53$ \\
Control group & 36 & $1.47 \pm 0.15$ & $2.18 \pm 0.21$ & $630.12 \pm 51.33$ & $4.03 \pm 0.27$ \\
F & $/$ & 4.763 & 5.431 & 13.884 & 6.162 \\
p & $/$ & $<0.05$ & $<0.05$ & $<0.05$ & $<0.05$ \\
\hline
\end{tabular}

TABLE 4: COMPARISON OF HOMA- $\beta$ AND HOMAIR INDEX IN THREE GROUPS

\begin{tabular}{lccc}
\hline Group & $\mathbf{n}$ & HOMA $-\mathbf{B}$ & HOMA-IR \\
\hline Non-obesity group & 36 & $7.81 \pm 0.23$ & $2.23 \pm 0.39$ \\
Obesity group & 64 & $5.45 \pm 0.11$ & $3.67 \pm 0.51$ \\
Control group & 36 & $9.14 \pm 0.36$ & $1.97 \pm 0.23$ \\
F & $/$ & 5.336 & 4.932 \\
p & $/$ & $<0.05$ & $<0.05$ \\
\hline
\end{tabular}

TABLE 5: CORRELATION ANALYSIS BETWEEN SERUM TG LEVEL AND GLUCOSE AND LIPID METABOLISM

\begin{tabular}{lcc}
\hline \multirow{2}{*}{ Test content } & \multicolumn{2}{c}{ TG } \\
\cline { 2 - 3 } & $\mathbf{r}$ & $\mathrm{p}$ \\
\hline HDL-C & 0.578 & 0.008 \\
LDL-C & 0.609 & 0.011 \\
FFA & 0.704 & 0.004 \\
TC & 0.727 & 0.001 \\
TG & -0.732 & 0.004 \\
GLU2 h & -0.618 & 0.015 \\
FPG & -0.677 & 0.008 \\
INS2 h & 0.609 & 0.012 \\
FINS & 0.611 & 0.031 \\
T & 0.564 & 0.022 \\
E2 & 0.802 & 0.001 \\
LH & 0.701 & 0.004 \\
FSH & 0.563 & 0.003 \\
\hline
\end{tabular}

evaluate obesity. Increasing studies have shown that hypertriglycemia is an independent risk factor for insulin resistance and serum TG content has also become an important indicator for clinical judgment of metabolic syndrome ${ }^{[15]}$. The mechanism of insulin resistance induced by hypertriglyceridemia may be that too much TG competes with glucose to enter cells, which has adverse effects on glucose oxidation and utilization. In addition, elevated TG is often accompanied by an increase in the level of its catabolic product FFA, which may interfere with the binding process of insulin with its receptor in tissues, resulting in the increase of insulin level in blood and the decrease of biological activity. Meanwhile, the increase of blood TG exceeds the body's ability to oxidize FFA and can lead to the accumulation of TG in liver, muscle, pancreas and other tissues. Finally, hypertriglyceridemia is often accompanied by overweight or obesity, especially abdominal obesity, which is the main manifestation of abnormal lipid metabolism affecting insulin sensitivity in Chinese ${ }^{[16]}$. In this study, the serum TG level of obesity group and non-obesity group was significantly higher than that of control group, the serum TG level of obesity group was significantly higher than that of non-obesity group and the more severe the obesity, the higher the serum TG level. Studies have shown that serum TG is related to glucose and lipid metabolism in patients with polycystic ovary syndrome ${ }^{[17,18]}$. Through data analysis, we found that serum TG level was directly related to glucose and lipid metabolism index, indicating that the glucose and lipid metabolism of patients can be judged by detecting serum TG level in patients. In conclusion, serum TG level in patients with PCOS can reflect the changes of glucose and lipid metabolism. As a cheap and routine test, TG can be considered as an alternative indicator of insulin resistance in PCOS non obesity patients.

\section{Acknowledgements:}

This work was supported by the Taizhou People's Hospital.

\section{Conflict and interests:}

The authors declared no conflict of interest.

\section{REFERENCES}

1. Norman RJ, Dewailly D, Legro RS, Hickey TE. Polycystic ovary syndrome. Lancet 2007;370:685-97.

2. Azziz R, Woods KS, Reyna R, Key TJ, Knochenhauer ES, Yildiz BO. The prevalence and features of the polycystic ovary syndrome in an unselected population. J Clin Endocrinol Metab 2004;89:2745-9.

3. Conway G, Dewailly D, Diamanti-Kandarakis E, EscobarMorreale HF, Franks S, Gambineri A, et al. The polycystic ovary syndrome: a position statement from the European Society of Endocrinology. Eur J Endocrinol 2014;171:P1-29.

4. Barber TM, McCarthy MI, Wass JA, Franks S. Obesity and polycystic ovary syndrome. Clin Endocrinol 2006;65:137-45.

5. Park SY, Cho YJ, Lee SR, Chung H, Jeong K. Triglyceride is a useful surrogate marker for insulin resistance in Korean women with polycystic ovary syndrome. Yonsei Med J 2015;56:785-92.

6. Kawamoto R, Kohara K, Kusunoki T, Tabara Y, Abe M, Miki $\mathrm{T}$. Alanine aminotransferase/aspartate aminotransferase ratio is the best surrogate marker for insulin resistance in non-obese Japanese adults. Cardiovasc Diabetol 2012;11:117. 
7. Bonora E, Targher G, Alberiche M, Bonadonna RC, Saggiani $\mathrm{F}$, Zenere MB, et al. Homeostasis model assessment closely mirrors the glucose clamp technique in the assessment of insulin sensitivity: studies in subjects with various degrees of glucose tolerance and insulin sensitivity. Diabetes care 2000;23:57-63.

8. Revised 2003 consensus on diagnostic criteria and long-term health risks related to polycystic ovary syndrome (PCOS). Hum Reprod 2004;19:41-7.

9. Murphy LJ. The role of the insulin-like growth factors and their binding proteins in glucose homeostasis. Exp Diabesity Res 2003;4:213-24.

10. Lee H, Oh JY, Sung YA. Adipokines, insulin-like growth factor binding protein-3 levels, and insulin sensitivity in women with polycystic ovary syndrome. Korean J Intern Med 2013;28:45663.

11. Carmina E, Legro RS, Stamets K, Lowell J, Lobo RA. Difference in body weight between American and Italian women with polycystic ovary syndrome: influence of the diet. Hum Reprod 2003;18:2289-93.

12. Valkenburg O, Steegers-Theunissen RP, Smedts HP, DallingaThie GM, Fauser BC, Westerveld EH, et al. A more atherogenic serum lipoprotein profile is present in women with polycystic ovary syndrome: a case-control study. J Clin Endocrinol Metab 2008;93:470-6.

13. Fruzzetti F, Perini D, Lazzarini V, Parrini D, Genazzani AR. Adolescent girls with polycystic ovary syndrome showing different phenotypes have a different metabolic profile associated with increasing androgen levels. Fertil Steril 2009;92:626-34.

14. Katz A, Nambi SS, Mather K, Baron AD, Follmann DA, Sullivan G, et al. Quantitative insulin sensitivity check index: a simple, accurate method for assessing insulin sensitivity in humans. J Clin Endocrinol Metab 2000;85:2402-10.

15. Kim-Dorner SJ, Deuster PA, Zeno SA, Remaley AT, Poth M. Should triglycerides and the triglycerides to high-density lipoprotein cholesterol ratio be used as surrogates for insulin resistance?. Metabolism 2010;59:299-304.

16. Wallace TM, Levy JC, Matthews DR. Use and abuse of HOMA modeling. Diabetes care 2004;27:1487-95.

17. Koschinsky ML. Novel insights into Lp (a) physiology and pathogenicity: more questions than answers?. Cardiovasc Hematol Disord Drug Targets 2006;6:267-78.

18. El-Mazny A, Abou-Salem N, El-Sherbiny W, El-Mazny A. Insulin resistance, dyslipidemia, and metabolic syndrome in women with polycystic ovary syndrome. Int J Gynaecol Obstet 2010;109:239-41..

This is an open access article distributed under the terms of the Creative Commons Attribution-NonCommercial-ShareAlike 3.0 License, which allows others to remix, tweak, and build upon the work non-commercially, as long as the author is credited and the new creations are licensed under the identical terms

This article was originally published in a special issue,
"Clinical Research in Pharmaceutical and Biomedical
Sciences" Indian J Pharm Sci 2021:83(1)Spl issue1;54-57 